\title{
Potential and pH Dependencies of Adsorbed Species of 2-, 4-Pyridinethiol and 2-Pyrimidinethiol on $\mathrm{Au}(111)$ Electrode
}

\author{
Isao TANIGUCHI, ${ }^{*}$ Soichiro Yoshimoto, Yukinari SUNATSUKI, \\ and Katsuhiko NISHIYAMA
}

Department of Applied Chemistry and Biochemistry, Faculty of Engineering, Kumamoto University (2-39-1, Kurokami, Kumamoto $860-8555$, Japan)

Received June 17, 1999 ; Accepted August 17, 1999

\begin{abstract}
Potential and $\mathrm{pH}$ dependencies of surface structures of $\mathrm{Au}(111)$ electrodes modified with 2- and 4-pyridinethiol (2-PySH and 4-PySH, respectively) and 2-pyrimidinethiol (2-PymSH) were examined by surface-enhanced IR absorption spectroscopy (SEIRAS) and STM. At positive potentials, the $p K a$ values of the adsorbed pyridinethiol and pyrimidine species decreased, and even in an acid solution the pyridine (or pyrimidine) $\mathrm{N}$ atom became less protonated. Therefore, STM images of self-assembled monolayers of these thiols observed at properly positive potentials (e.g., >ca. $0.3 \mathrm{~V}$ vs. $\mathrm{Ag} / \mathrm{AgCl}$ for $2-\mathrm{PySH}$ and $2-\mathrm{PymSH}$ in a $0.1 \mathrm{M} \mathrm{HClO}_{4}$ solution, and $>0.7 \mathrm{~V}$ vs. $\mathrm{Ag} /$ $\mathrm{AgCl}$ for 4 - $\mathrm{PySH}$ in a $0.01 \mathrm{M}\left(\mathrm{M}=\mathrm{mol} \mathrm{dm} \mathrm{dm}^{-3}\right) \mathrm{HClO}_{4}$ solution) can be used for evaluating the structures in a neutral solution.
\end{abstract}

Key Words : Self-assembled Monolayer (SAM), Single Crystal Electrode, SEIRAS, STM

\section{Introduction}

In recent years, various functional electrodes for metalloprotein electrochemistry have been developed. ") For cytochrome c electrochemistry, gold surfaces modified with self-assembled monolayers of electron-transfer promoters such as bis(4-pyridyl) disulfide (4,4'-PySSPy) and 4-pyridinethiol (4-PySH) are known to be effective., ${ }^{1,2)}$ Using atomically flat $\mathrm{Au}$ single crystal surfaces, electrochemical responses of cytochrome $c$ were enhanced at electrodes modified with 4,4'-PySSPy or 4-PySH, but not at the surfaces modified with bis(2-pyridyl)disulfide (2,2'-PySSPy) or 2-pyridinethiol (2-PySH). ${ }^{3}$ ) The Au(111) electrodes having an enough surface area for spectroelectrochemical measurements can be easily prepared by using the vacuum evaporation technique, although when Au(111) surfaces modified with 4-PySH are used, a small amount of sulfide impurity in a modifier solution should be taken into account. ${ }^{4-7)}$

In the present study, potential and $\mathrm{pH}$ dependencies of surface structures of 2-, 4-PySH and 2pyrimidinethiol (2-PymSH) modified Au(111) electrodes were examined by surface-enhanced IR absorption spectroscopy (SEIRAS) and STM.

\section{Experimental}

$\mathrm{Au}$ (111) single crystals were prepared by the flameannealing-quenching method developed by Clavilier et al. ${ }^{8)}$ Details for the preparation of $\mathrm{Au}(111)$ single crystal electrodes and for the surface modification were the same as those described elsewhere. ${ }^{3,6,7)}$

SEIRAS coupled with attenuated-total-reflection ${ }^{9)}$ of the adsorbed thiols on $\mathrm{Au}(111)$ surfaces was carried out using a Bio-Rad FTS-6000 IR spectrometer with a
HgCdTe (MCT) detector at the resolution of $4 \mathrm{~cm}^{-1}$ and a home-made spectroelectrochemical cell. The working electrode was vacuum-evaporated Au(111)oriented thin ( $c a .20 \mathrm{~nm}$ thick) film on the basal plane of a semi-cylindrical Si prism. In situ electrochemical STM measurements of thiol monolayers were carried out in a $0.05 \mathrm{M} \mathrm{HClO}_{4}$ solution using a Nanoscope $\mathrm{E}$ and an electrochemically etched tungsten tip. ${ }^{10)}$ The typical potentials of the sample and tip used were 0.8 and $0.5 \mathrm{~V}$ vs. RHE, respectively. The tunneling current used was $2 \mathrm{nA}$. Cyclic voltammetry at an $\mathrm{Au}(111)$ single crystal electrode was carried out at $25{ }^{\circ} \mathrm{C}$ using the meniscus (or hanging electrolyte) method. Platinum plate $(1.5 \times 0.7 \mathrm{~cm})$ was used as a counter electrode. $\mathrm{An} \mathrm{Ag} / \mathrm{AgCl}$ (saturated $\mathrm{KCl}$ ) was used as a reference electrode.

2-PySH (Aldrich, $99 \%$ ) and 2-PymSH (Aldrich, 98 $\%$ ) were used as received, while 4-PySH (Aldrich, 95 $\%$ ) was purified by anion exchange chromatography using a DE-52 column to remove sulfide impurity.,7)

\section{Results and Discussion}

A 4-PySH modified Au(111) electrode gave a welldefined voltammetric response of cytochrome $c$, while no electrode reaction of cytochrome $c$ took place at a 2-PySH modified electrode. 2-PymSH was also effective to promote the electron transfer reaction of cytochrome c. Similar results were also observed when corresponding disulfides instead of these thiols were used as electrode modifiers.

To understand the surface structures of these modified electrodes more precisely at the molecular level, in situ electrochemical SEIRA spectra of $\mathrm{Au}(111)$ surfaces modified with 2- and 4-PySH, and 
2-PymSH were measured. The SEIRA spectra of adsorbed thiols depended on $\mathrm{pH}$ of the solution as shown in Fig. 1. From the IR spectra of both thiols and corresponding disulfides (see Fig. 1), IR signals for protonated species can be assigned, because thiols have thione-thiol tautomerism (i.e., $\mathrm{PySH}=\mathrm{SPyH}$ ) and the thione form ( $\mathrm{SPyH}$ ) gives similar signals to those of protonated species.

For a 4-PySH modified electrode (Fig. 1A), in an alkaline solution unprotonated 4 -PySH on the electrode, Au-SPy, gave peaks at 1578 and $1476 \mathrm{~cm}^{-1}$ (assigned to be the $8 \mathrm{a}$ and $19 \mathrm{a}$ modes, respectively), while in an acidic solution protonated PySH adsorbed on the electrode, Au-SPyH ${ }^{+}$, gave corresponding peaks at 1620 and $1467 \mathrm{~cm}^{-1}$. Both of these signals are seen in a $0.1 \mathrm{M} \mathrm{NaClO}_{4}$ solution. The SEIRA spectra of the 2-PySH modified Au(111) surface showed signals at 1578 and $1448 \mathrm{~cm}^{-1}$ (as the $8 \mathrm{a}$ and 19a modes, respectively), and only a small peak at $1606 \mathrm{~cm}^{-1}(8 \mathrm{a}$ mode) for the protonated species was seen in an acidic solution (Fig. 1B). Similar results (signals at 1566 for 8 a mode and $1377 \mathrm{~cm}^{-1}$ for $19 \mathrm{a}$ mode) were also

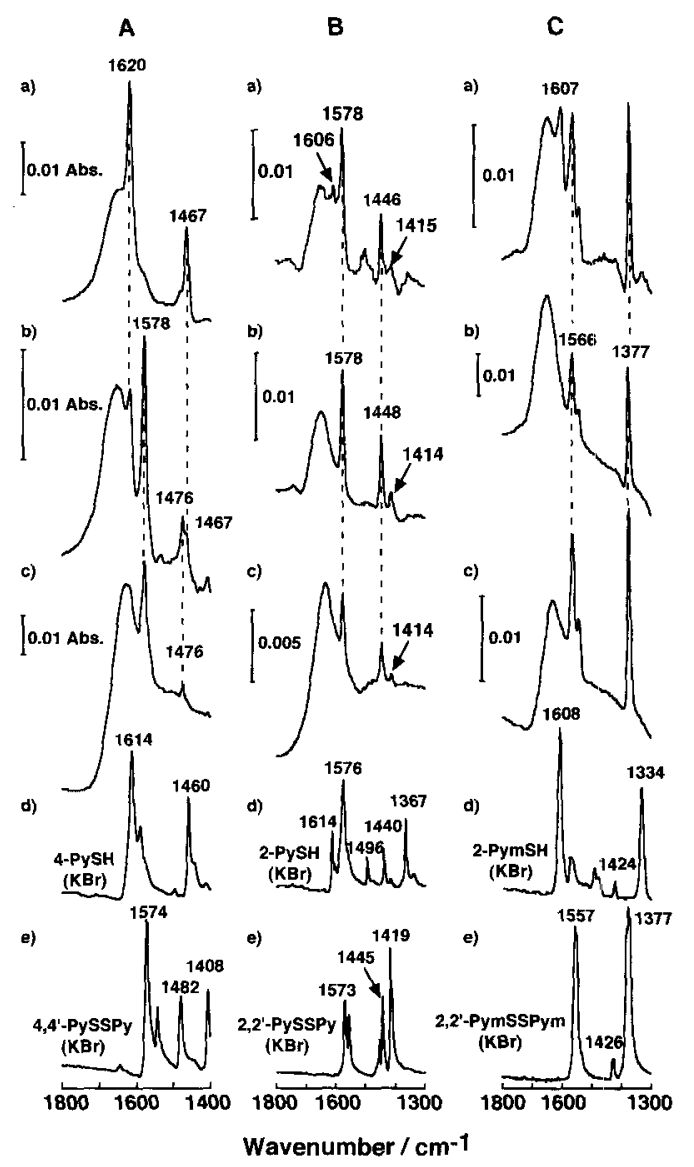

Fig. 1 Typical SEIRA spectra of A) 4- and B) 2-PySH and C) 2-PymSH adsorbed on an $\mathrm{Au}(111)$-oriented film electrode at $0 \mathrm{~V}$ vs. $\mathrm{Ag} / \mathrm{AgCl}$ (sat. $\mathrm{KCl}$ ) in a) $0.1 \mathrm{M} \mathrm{HClO}_{4}$, b) $0.1 \mathrm{M} \mathrm{NaClO}_{4}$, and c) $0.1 \mathrm{M} \mathrm{NaOH}$ solutions, together with IR spectra for d) thiols and e) corresponding disulfides obtained by the $\mathrm{KBr}$ technique. IR signals were given with broad peak due to the surface water molecules. Data were accumulated 256 times for each spectrum. observed for the 2-PymSH modified surface (Fig. 1C). Again, a small peak at $1607 \mathrm{~cm}^{-1}$ (8a mode) was seen for the protonated species $\left(\mathrm{Au}-\mathrm{SPymH}^{+}\right)$in an acidic solution.

These results are reasonable because the $p K a$ values at the pyridine and pyrimidine $\mathrm{N}$ of 2-PySH and 2 -PymSH are reported to be smaller ${ }^{11)}$ than that of 4 -PySH. The surface $p K a$ value $\left(=4.6^{12)} ; 3.9^{13)}\right)$ at the pyridine $\mathrm{N}$ of 4-PySH adsorbed on an $\mathrm{Au}(111)$ electrode has been reported to shift by $c a .3 \mathrm{pH}$ units from that $\left(p K a=1.43^{11)}\right)$ in solution.

Interestingly, at positive potentials (e.g.,>ca. $0.3 \mathrm{~V}$ vs. $\mathrm{Ag} / \mathrm{AgCl}$, SEIRA spectra in a $0.1 \mathrm{M} \mathrm{HClO}_{4}$ solution were almost the same as those obtained in neutral and alkaline solutions at $0 \mathrm{~V}$ ( $c f$. Figs. 1 and 2 for 2-PySH and 2-PymSH). Similar results were also seen for 4 -PySH (at>ca. $0.7 \mathrm{~V}$ vs. $\mathrm{Ag} / \mathrm{AgCl}$ in a $0.01 \mathrm{M}$ $\mathrm{HClO}_{4}$ solution, not shown). At potentials more negative than $-0.2 \mathrm{~V}$ vs. $\mathrm{Ag} / \mathrm{AgCl}$ (see Fig. 2 at $-0.2 \mathrm{~V}$ ), signals due to protonated species became predominant. This means that at positive potentials the pyridine (or pyrimidine) $\mathrm{N}$ atom became less protonated. This is important because STM images obtained in an acid solution at a positive potential ${ }^{10,14)}$ would give the structure of unprotonated species of the modifier adsorbed on the electrode, like in a neutral solution in which metalloprotein electrochemistry has usually been carried out.

In situ electrochemical STM measurements of the $\mathrm{Au}(111)$ facet surfaces modified with thiols gave the following results (Fig. 3). The 4-PySH modified electrode showed the rectangular unit cell of $p(5 \times \sqrt{3} R-$

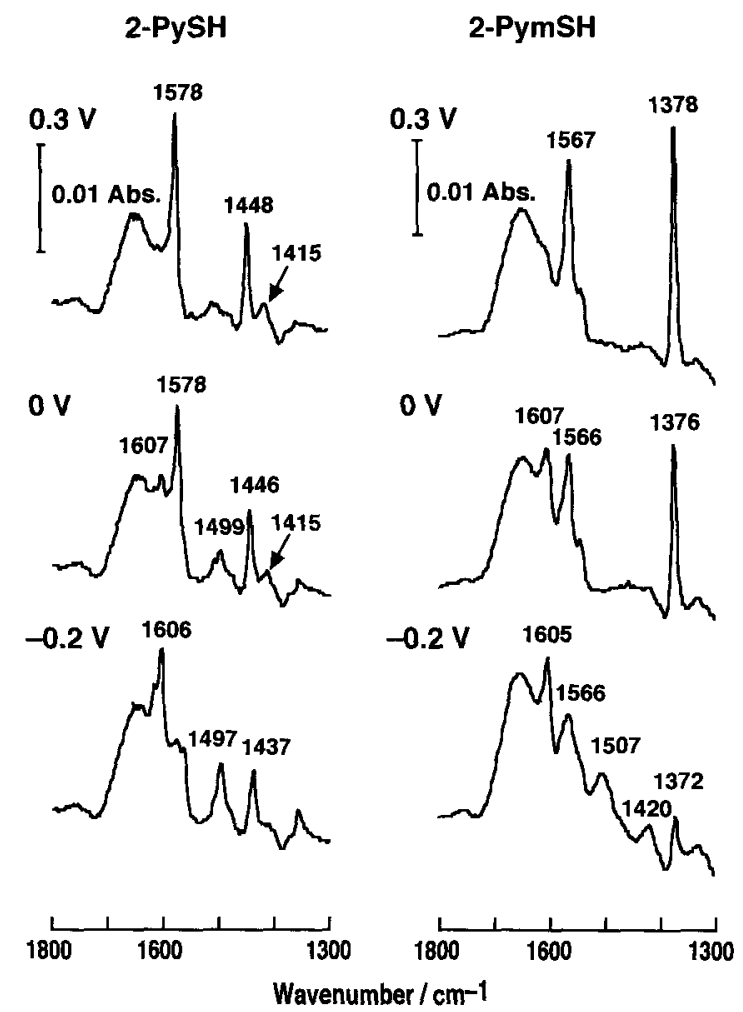

Fig. 2 Typical SEIRA spectra of 2-PySH and 2-PymSH adsorbed on an $\mathrm{Au}(111)$-oriented film electrode in a $0.1 \mathrm{M}$ $\mathrm{HClO}_{4}$ solution at various potentials. 
$30^{\circ}$ ) structure with an interaction of two neighboring 4-PySH. ${ }^{10,15)}$ On the other hand, 2-PySH modified electrode showed that the intermolecular spacing of $0.78 \mathrm{~nm}$ for the $\sqrt{7}$ direction and $1.2 \mathrm{~nm}$ for the $\mathrm{Au}(111)$ atomic row, which can be given as a $p\left(4 \times \sqrt{7} R-40.9^{\circ}\right)$ structure with no dimer formation. ${ }^{14)}$ The STM images suggested that 2-PySH adsorbed both at thiolate $\mathrm{S}$ and pyridine $\mathrm{N}$ atoms on the electrode surface. The STM image of 2-PymSH on $\mathrm{Au}(111)$ was similar to that of 2-PySH, given as a $p(5 \times \sqrt{7}$ $R-40.9^{\circ}$ ), but the suggested structure is not the same as that of 2 -PymSH in the air. ${ }^{16)}$

The surface excesses $(5.0 \pm 0.2,4.7 \pm 0.2$, and $4.8 \pm$ $0.2 \times 10^{-10}$ moles $\mathrm{cm}^{-2}$ for 4 -PySH, 2 -PySH and 2 PymSH, respectively) estimated from the peak areas for the reductive desorption of thiols from the Au(111) surface in a $0.1 \mathrm{M} \mathrm{KOH}$ solution (e.g., Au-SPy $+\mathrm{e}^{-}=$ $\mathrm{Au}+\mathrm{PyS}^{-}$) were in good agreement with those calculated for the proposed structures from the STM images.

Since the IR signal due to the $b_{1}$ mode was seen for unprotonated 2-PySH on the electrode at ca. 1415 $\mathrm{cm}^{-1}$, pyridine ring plane would be perpendicular to the electrode surface. On the other hand, no significant signal due to the $b_{1}$ mode was seen for the electrode surfaces modified with unprotonated 2PymSH and 4-PySH, for these molecules pyridine and
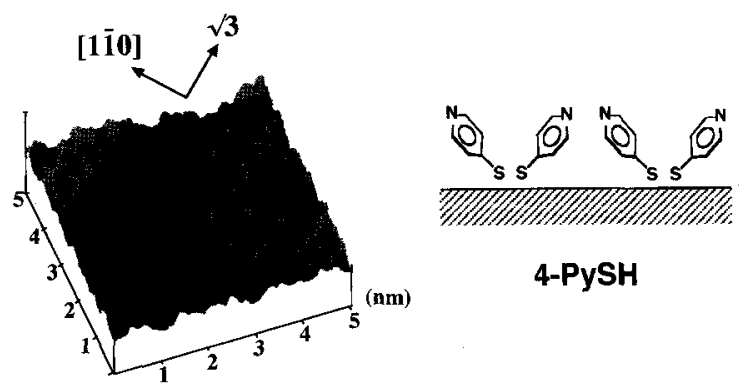

4-PySH
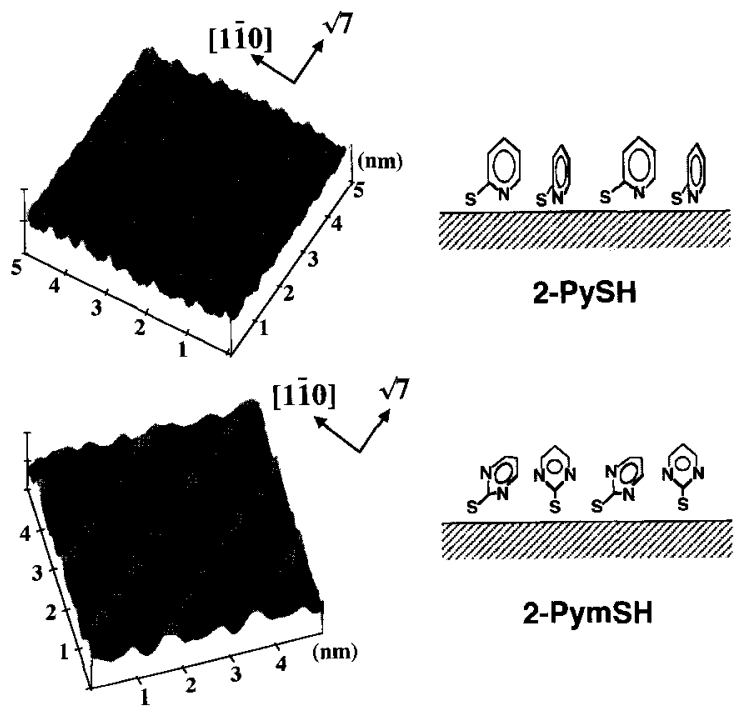

2-PymSH

Fig. 3 Typical STM images with rectangular unit cells and schematic illustrations of possible surface structures of Au(111) electrodes modified with 2- and 4-PySH and 2-PymSH. See the text for experimental conditions of STM measurements. pyrimidine ring plane would not be oriented perpendicular to the electrode surface but rather parallel. Taking into account the STM images and the SEIRA spectra observed, possible surface structures of these modified electrodes in a neutral solution are given in Fig. 3. The suggested surface structures are consistent with the idea that the pyridine or pyrimidine $\mathrm{N}$ atom is required to be directed to the solution for the well-defined electrochemical responses of cytochrome c.

\section{Acknowledgment}

This work was supported in part by a Grant-in-Aid from the Ministry of Education, Science, Sports and Culture, Japan (No. 11450329) and that for Scientific Research on Priority Area of Electrochemistry of Ordered Interfaces (No. 09237106).

\section{References}

1) F. M. Hawkridge and I. Taniguchi, Comments on Inorg. Chem., 17, 163 (1995); I. Taniguchi, Interface, 4, 34 (1997) and references therein.

2) I. Taniguchi, K. Toyosawa, H. Yamaguchi, and K. Yasukouchi, J. Chem. Soc. Chem. Commun., 1982, 1032; J. Electroanal. Chem., 140, 187 (1982).

3) I. Taniguchi, S. Yoshimoto, and K. Nishiyama, Chem. Lett., 1997, 353.

4) B. D. Lamp, D. Hobara, M. D. Porter, K. Niki, and T. M. Cotton, Langmuir, 13, 736 (1997); C-J. Zhong, R. C. Brush, J. Anderegg, and M. D. Porter, Langmuir, 15, 518 (1999).

5) I. Taniguchi, S. Yoshimoto, M. Yoshida, Y. Mie, K. Kudo, and K. Nishiyama, Novel Trends in Electroorganic Synthesis, (Ed. S. Torii), Springer-Verlag, Tokyo p. 175 (1998).

6) I. Taniguchi, S. Yoshimoto, M. Yoshida, S. Kobayashi, T. Miyawaki, Y. Aono, Y. Sunatsuki, and H. Taira, Electrochim. Acta, in press (1999).

7) S. Yoshimoto, M. Yoshida, S. Kobayashi, S. Nozute, T. Miyawaki, Y. Hashimoto, and I. Taniguchi, J. Electroanal. Chem., 473, 85 (1999).

8) J. Clavilier, R. Faure, G. Guinet, and R. Durand, J. Electroanal. Chem., 107, 205 (1980).

9) M. Osawa, Bull. Chem. Soc., Jpn. 70, 2861 (1997).

10) T. Sawaguchi, F. Mizutani, and I. Taniguchi, Langmuir, 14, 3565 (1998).

11) Lange's Handbook of Chemistry, 13th edition, (Ed. J. A. Dean), MacGraw-Hill Book Company, New York, N. Y., p. 5-46. (1985).

12) M. A. Bryant and R. M. Crooks, Langmuir, 9, 385 (1993).

13) H.-Z. Yu, N. Xia, and Z.-F. Liu, Anal. Chem., 71, 1354 (1999).

14) T. Sawaguchi, S. Yoshimoto, F. Mizutani, and I. Taniguchi, Electrochim. Acta, in press (1999).

15) L.-J. Wan, Y. Hara, H. Noda, and M. Osawa, J. Phys. Chem. B, 102, 5943 (1998).

16) J. J. Davis, H. A. O. Hill, R. Yamada, H. Naohara, and K. Uosaki, J. Chem. Soc., Faraday Trans., 94, 1315 (1998). 